\title{
A Method for Fault Prediction of Air Brake System in Vehicles
}

\author{
Hossam A. Nabwey ${ }^{1,2}$, Abdulfattah Omar $^{3}$ \\ ${ }^{1}$ Department of Mathematics, College of Science and Humanities in Al-Kharj, Prince Sattam bin Abdulaziz University, Al-Kharj11942, Saudi Arabia. \\ ${ }^{2}$ Department of Basic Engineering Science, Faculty of Engineering, Menoufia University, Shebin El-Kom, 32511, Egypt. \\ ${ }^{3}$ Department of English, College of Science and Humanities in Al-Kharj, Prince Sattam bin Abdulaziz University, Al-Kharj11942, Saudi Arabia.
}

${ }^{3}$ https://orcid.org/0000-0002-7167-3822

\begin{abstract}
Fault diagnosis is a complex problem that concerns effective decision-making. Air brake system is a crucial safety unit and failure of which leads to the loss of vehicle yaw stability and increase in stopping distance of the vehicle. Fault detection and isolation in brake system is critical for continuity of the performance and the safe running of autonomous vehicles. Carrying out timely system diagnosis whenever a fault occur is important to prevent component degradation and vehicle breakdown. This work use the Rough Set Theory to develop fault diagnostic scheme for classifying the "fault" and "Nofault" conditions of air brake system with the knowledge of wheel speed sensor data. The rough set reduction principle is applied to find all reducts, and then a set of generalized classification rules for predicting the faults of Air brake system was extracted. The results show that the presented method can effectively enrich the vehicle condition monitoring system, able to give a feedback to the driver regarding the working condition of air brake system, detect and identify the location of faults, minimize the resources, such as time, cost of maintenance, etc.
\end{abstract}

Keywords: Classification ; Rules Extraction ; Air Brake ; Fault Diagnosis;; Feature Selection; Rough Set Theory; Wheel Speed Sensor; decision making

\section{INTRODUCTION}

Fault diagnosis in brake system is significant for improving the performance of autonomous vehicles and advanced driver assistance systems where it detecting dangerous situations and react accordingly in order to avoid or mitigate accidents. A fault diagnosis system can enable continuous and reliable operation of vehicular systems through effective vehicle health monitoring. In Vehicles, air brake system is a critical safety unit and failure of which results in not only the loss of vehicle yaw stability, but also an increase in stopping distance of the vehicle. Hence, brake faults may even lead to severe road safety issues and accidents.

One of the reasons for air brake fault is the increase in length of a component called pushrod beyond readjustment limit. The classical method for safety inspection of pushrod stroke measurement requires a human being to go underneath the vehicle and manually measure the pushrod stroke. It is a timeconsuming procedure and difficult if the vehicle ground clearance is low. So the need for developing an intelligent diagnostic scheme to minimize the resources (such as time and cost of maintenance) becomes an urgent research subject currently. Recently, model-based and data-driven approaches are used in fault diagnostic schemes [1-4]. To inspect the health conditions of air brake system, condition monitoring systems are used to collect real-time data from them, and big data are acquired due to development of the Internet, the internet of things, wireless communications, mobile devices, and smart manufacturing of sensors, so the amount of data collected has grown in an exponential manner which leads the task of fault diagnosis has become increasingly difficult and its complexity almost unmanageable using traditional techniques.

Machine learning techniques like Artificial Neural Network, Support Vector Machines and Decision tree are used in a wide variety of classification algorithms for fault diagnosis. Rough set theory (RST) [5, 6] which is one of the successful approximations based mathematical model to deal the imprecision and uncertainty present in knowledge. Many heuristic algorithms are proposed based on rough set theory, also numerous approached based on rough set theory and other theories are investigated to extract decision rules and reduce the dimensionality of dataset [7-26]. One advantage of the rough set is the creation of readable if-then rules. Such rules have a potential to reveal new patterns in the data material. Thus, the main objective of this work is to present a Method based on Rough Set Theory to develop fault diagnostic scheme for classifying the "fault" and "No-fault" conditions of air brake system with the knowledge of wheel speed sensor data.

\section{AIR BRAKE SYSTEM}

Air brake system is a type of braking system generally used in heavy commercial vehicles or vehicles which require some really powerful and efficient braking system. It is a kind of friction brake where instead of hydraulic-fluid; air is used as the compression media for brake pads. Application of air brakes becomes a necessity in case of trucks having multiple trailers, high-speed long-haul buses, vehicles of military utility and semi-trailers. Air brakes were invented by George Westinghouse for use in trains. After having proved its caliber in trains, air brakes were later adapted to be used in heavy vehicles. The safety and braking confidence that air brakes provide to heavy vehicles are vouched for till day [27]. 
This section will explain how the Air Brake System Work. At the begging fig.1 shows Basic Air Brake System when the brakes are not applied. When the driver of a vehicle presses the brake pedal in order to stop or decelerate the vehicle the following processes takes place [28]:

- When the driver starts the engine the brake compressor starts as it is driven by the engine which in turn starts compressing the atmospheric air and through the compressor governor this compressed air with optimum pressure is sent to the compressed air reservoir which always has some amount of air stored from the previous cycle.

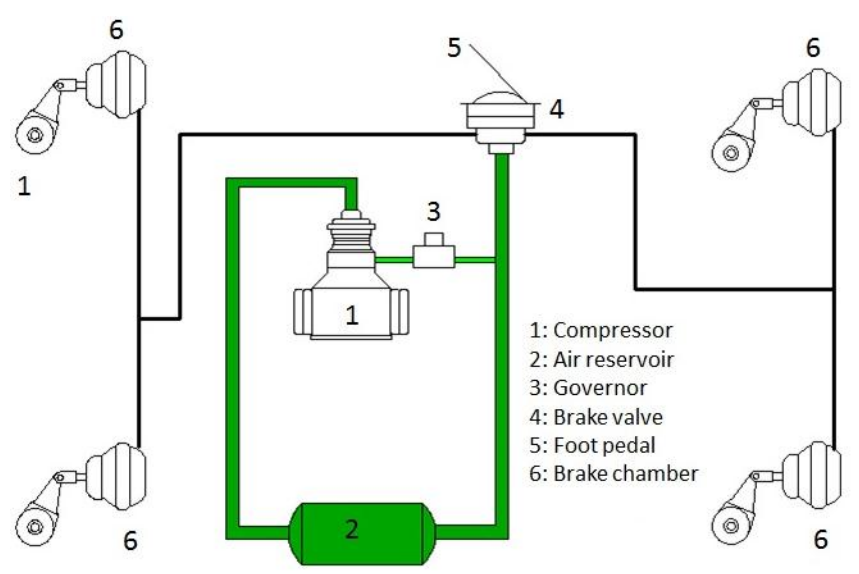

Fig. 1.Basic Air Brake System when the brakes are not applied

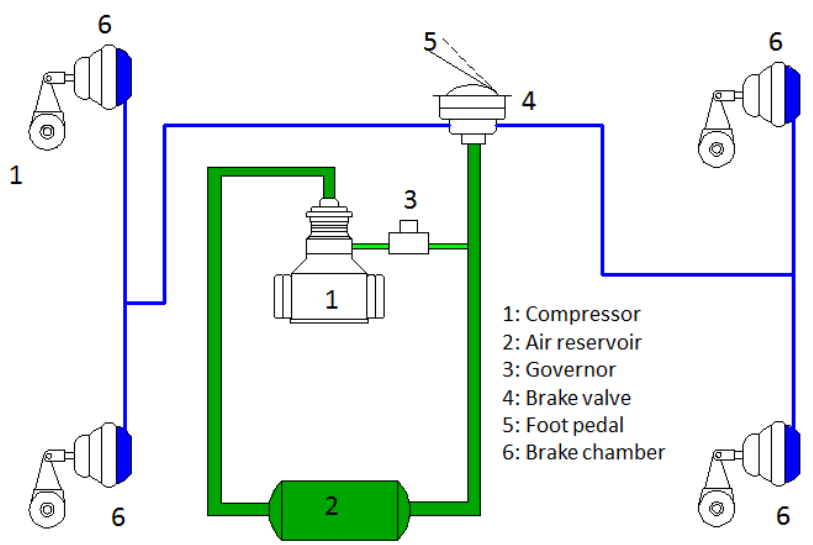

Fig. 2.Basic Air Brake System when the brakes are applied

- When the driver presses the brake pedal the outlet valve of the triple valve closes and inlet valve opens up which in turn gives passage to the compressed air from the reservoir to pass through the brake lines of the system.

- This compressed air flowing through the brake lines is then transferred to the brake cylinder which has piston inside it.

- When the compressed air applies pressure over the piston inside the brake chamber, piston moves away from its original position which converts this pneumatic energy into the mechanical energy.

- On the wheel end of the brake cylinder, brake drums are placed inside which there is a housing of the mechanical actuator like springs or slacks having brake pads at its outer end.

- Due to the movement of piston because of the pressure applied by the compressed air, the mechanical actuator inside the brake drum expands which in turn pushes the brake pads in outward direction in order to make frictional contact with the rotating drum lines.

- With this frictional contact between brake pads and rotating drum lines brakes are applied to the wheels in order to stop or decelerate the vehicle.

\section{PROPOSED METHODOLOG}

The wheel speed sensor is a basic component of Anti-lock Brake System (ABS), which has been made mandatory in Heavy Commercial Road Vehicles in many countries. It can be used for fault diagnosis. Hence this paper propose a Method based on Rough Set Theory to develop fault diagnostic scheme for classifying the "fault" and "No-fault" conditions of air brake system with the knowledge of wheel speed sensor data. The decision table shown in Table 1 was developed by [29] to represent the fault diagnostic schemes for front right brake considering the fault / no-fault conditions of other brakes (front left, rear left, rear right). It is also noting that the same training data sets can be used for developing fault diagnostic schemes for other brakes with corresponding change in the labeling, but here we will concentrate to explain the fault diagnostic scheme for only the front right brake.

TABLE I. DECISION TABLE FOR FAULT DETECTION AND ISOLATION OF FRONT RIGHT BRAKE CHAMBER

\begin{tabular}{|c|c|c|c|c|c||}
\hline U & $\begin{array}{c}\text { front } \\
\text { right }\end{array}$ & $\begin{array}{c}\text { front } \\
\text { left }\end{array}$ & $\begin{array}{c}\text { Rear } \\
\text { right }\end{array}$ & $\begin{array}{c}\text { Rear } \\
\text { left }\end{array}$ & Decision \\
\hline $\mathrm{X} 1$ & No & No & No & No & $\begin{array}{c}\text { No Fault in front right } \\
\text { brake }\end{array}$ \\
\hline $\mathrm{X} 2$ & Yes & No & No & No & $\begin{array}{c}\text { Fault in front right } \\
\text { brake }\end{array}$ \\
\hline $\mathrm{X} 3$ & No & Yes & No & No & $\begin{array}{c}\text { No Fault in front right } \\
\text { brake }\end{array}$ \\
\hline $\mathrm{X} 4$ & Yes & Yes & No & No & $\begin{array}{c}\text { Fault in front right } \\
\text { brake }\end{array}$ \\
\hline $\mathrm{X} 5$ & No & No & Yes & No & $\begin{array}{c}\text { No Fault in front right } \\
\text { brake }\end{array}$ \\
\hline $\mathrm{X} 6$ & Yes & No & Yes & No & $\begin{array}{c}\text { Fault in front right } \\
\text { brake }\end{array}$ \\
\hline $\mathrm{X} 7$ & No & No & No & Yes & $\begin{array}{c}\text { No Fault in front right } \\
\text { brake }\end{array}$ \\
\hline $\mathrm{X} 8$ & Yes & No & No & Yes & $\begin{array}{c}\text { Fault in front right } \\
\text { brake }\end{array}$ \\
\hline
\end{tabular}


International Journal of Engineering Research and Technology. ISSN 0974-3154, Volume 13, Number 5 (2020), pp. 1002-1008

(C) International Research Publication House. https://dx.doi.org/10.37624/IJERT/13.5.2020.1002-1008

\begin{tabular}{||c|c|c|c|c|c||}
\hline X9 & No & Yes & Yes & No & $\begin{array}{c}\text { No Fault in front right } \\
\text { brake }\end{array}$ \\
\hline X10 & Yes & Yes & Yes & No & $\begin{array}{c}\text { Fault in front right } \\
\text { brake }\end{array}$ \\
\hline X11 & No & No & Yes & Yes & $\begin{array}{c}\text { No Fault in front right } \\
\text { brake }\end{array}$ \\
\hline X12 & Yes & No & Yes & Yes & $\begin{array}{c}\text { Fault in front right } \\
\text { brake }\end{array}$ \\
\hline X13 & No & Yes & No & Yes & $\begin{array}{c}\text { No Fault in front right } \\
\text { brake }\end{array}$ \\
\hline X14 & Yes & Yes & No & Yes & $\begin{array}{c}\text { Fault in front right } \\
\text { brake }\end{array}$ \\
\hline X15 & No & Yes & Yes & Yes & $\begin{array}{c}\text { No Fault in front right } \\
\text { brake }\end{array}$ \\
\hline X16 & Yes & Yes & Yes & Yes & $\begin{array}{c}\text { Fault in front right } \\
\text { brake }\end{array}$ \\
\hline
\end{tabular}

When a boundary region exists; i.e. when $\bar{R} X-\underline{R} X \neq 0$ then $\mathrm{X}$ is a Rough Set

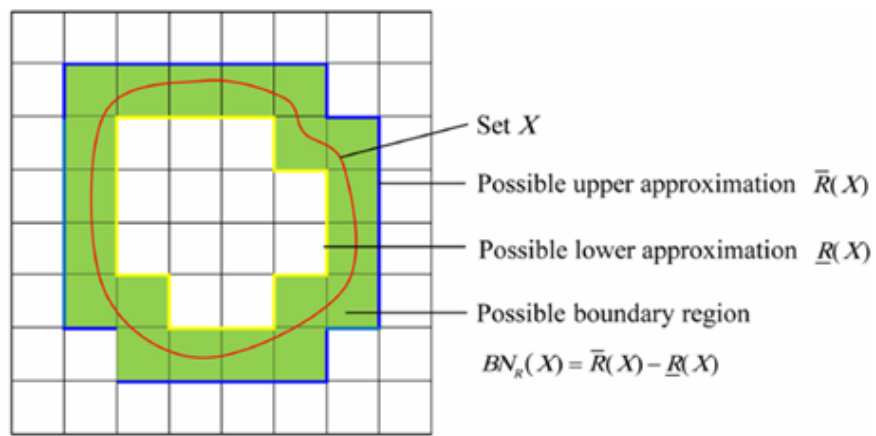

Fig. 3.Representation of Upper approximation and Lower approximation

Now, we will discuss the proposed method to analyze, mining and generating diagnostic rules based on rough sets theory which constitutes a sound basis for KDD. It offers mathematical tools to discover patterns hidden in data and can be used for feature selection, feature extraction, data reduction, decision rule generation, and pattern extraction. The main concepts of rough sets can be summarized as follow:

\section{A. Indiscernibility relations}

Indiscernibility is central to the rough sets and the objects in a decision table are classified into equivalent classes which are called concepts. An indiscernible relation $\operatorname{IND}(B)$ can be defined as:

$$
I N D(B)=\{(\mathrm{x}, \mathrm{y}) \in \mathrm{U} \mid \forall \mathrm{a} \in \mathrm{B}, \mathrm{a}(\mathrm{x})=\mathrm{a}(\mathrm{y})\}
$$

\section{B. The Lower Approximation of $X$}

is the set contain all objects which with certainty belong to the set $\mathrm{X}$. it can be defined as:

$$
\underline{R} X=\bigcup_{x \in U}\{R(x): R(x) \subseteq X\}
$$

\section{The Upper Approximation of $X$}

is the set contain all objects which possibly belong to the set $X$. it can be defined as:

$$
\bar{R} X=\bigcup_{x \in U}\{R(x): R(x) \cap X \neq \phi\}
$$

\section{The Boundary Region of $X$}

is the difference between upper and lower approximation as shown in fig.3. it can be defined as :

$$
B N_{R}(X)=\bar{R} X-\underline{R} X
$$

Now by considering the decision table in Table 2, we can find Indiscernibility relations as follow:

$$
\begin{aligned}
& I N D(\{\text { front right }\})=\left\{\begin{array}{l}
\{\mathrm{X} 1, \mathrm{X} 3, \mathrm{X} 5, \mathrm{X} 7, \mathrm{X} 9, \mathrm{X} 11, \mathrm{X} 13, \mathrm{X} 15\}, \\
\{\mathrm{X} 2, \mathrm{X} 4, \mathrm{X} 6, \mathrm{X} 8, \mathrm{X} 10, \mathrm{X} 12, \mathrm{X} 14, \mathrm{X} 16\}
\end{array}\right\} \\
& I N D(\{\text { front left }\})=\left\{\begin{array}{l}
\{\mathrm{X} 1, \mathrm{X} 2, \mathrm{X} 5, \mathrm{X} 6, \mathrm{X} 7, \mathrm{X} 8, \mathrm{X} 11, \mathrm{X} 12\}, \\
\{\mathrm{X} 3, \mathrm{X} 4, \mathrm{X} 9, \mathrm{X} 10, \mathrm{X} 13, \mathrm{X} 14, \mathrm{X} 15, \mathrm{X} 16\}
\end{array}\right\}
\end{aligned}
$$

$I N D(\{$ Rear right $\})=\left\{\begin{array}{l}\{\mathrm{X} 1, \mathrm{X} 2, \mathrm{X} 3, \mathrm{X} 4, \mathrm{X} 7, \mathrm{X} 8, \mathrm{X} 13, \mathrm{X} 14\}, \\ \{\mathrm{X} 5, \mathrm{X} 6, \mathrm{X} 9, \mathrm{X} 10, \mathrm{X} 11, \mathrm{X} 12, \mathrm{X} 15, \mathrm{X} 16\}\end{array}\right\}$

$I N D(\{$ Rear left $\})=\left\{\begin{array}{l}\{\mathrm{X} 1, \mathrm{X} 2, \mathrm{X} 3, \mathrm{X} 4, \mathrm{X} 5, \mathrm{X} 6, \mathrm{X} 9, \mathrm{X} 10\}, \\ \{\mathrm{X} 7, \mathrm{X} 8, \mathrm{X} 11, \mathrm{X} 12, \mathrm{X} 13, \mathrm{X} 14, \mathrm{X} 15, \mathrm{X} 16\}\end{array}\right\}$

$I N D(\{$ front right,front left $\})=\left\{\begin{array}{l}\{\mathrm{X} 1, \mathrm{X} 5, \mathrm{X} 7, \mathrm{X} 11\},\{\mathrm{X} 3, \mathrm{X} 9, \mathrm{X} 13, \mathrm{X} 15\} \\ \{\mathrm{X} 2, \mathrm{X} 6, \mathrm{X} 8, \mathrm{X} 12\},\{\mathrm{X} 4, \mathrm{X} 10, \mathrm{X} 14, \mathrm{X} 16\}\end{array}\right\}$

$I N D(\{$ front right,Rear right $\})=\left\{\begin{array}{l}\{\mathrm{X} 1, \mathrm{X} 3, \mathrm{X} 7, \mathrm{X} 13\},\{\mathrm{X} 5, \mathrm{X} 9, \mathrm{X} 11, \mathrm{X} 15\}, \\ \{\mathrm{X} 2, \mathrm{X} 4, \mathrm{X} 8, \mathrm{X} 14\},\{\mathrm{X} 6, \mathrm{X} 10, \mathrm{X} 12, \mathrm{X} 16\}\end{array}\right\}$

$I N D(\{$ front right,Rear left $\})=\left\{\begin{array}{l}\{\mathrm{X} 1, \mathrm{X} 3, \mathrm{X} 5, \mathrm{X} 9\},\{\mathrm{X} 7, \mathrm{X} 11, \mathrm{X} 13, \mathrm{X} 15\}, \\ \{\mathrm{X} 2, \mathrm{X} 4, \mathrm{X} 6, \mathrm{X} 10\},\{\mathrm{X} 8, \mathrm{X} 12, \mathrm{X} 14, \mathrm{X} 16\}\end{array}\right\}$

$I N D(\{$ front left,Rear right $\})=\left\{\begin{array}{l}\{\mathrm{X} 1, \mathrm{X} 2, \mathrm{X} 7, \mathrm{X} 8\},\{\mathrm{X} 5, \mathrm{X} 6, \mathrm{X} 11, \mathrm{X} 12\}, \\ \{\mathrm{X} 3, \mathrm{X} 4, \mathrm{X} 13, \mathrm{X} 14\},\{\mathrm{X} 9, \mathrm{X} 10, \mathrm{X} 15, \mathrm{X} 16\}\end{array}\right\}$

$I N D(\{$ front left,Rear left $\})=\left\{\begin{array}{l}\{\mathrm{X} 1, \mathrm{X} 2, \mathrm{X} 5, \mathrm{X} 6\},\{\mathrm{X} 7, \mathrm{X} 8, \mathrm{X} 11, \mathrm{X} 12\}, \\ \{\mathrm{X} 3, \mathrm{X} 4, \mathrm{X} 9, \mathrm{X} 10\},\{\mathrm{X} 13, \mathrm{X} 14, \mathrm{X} 15, \mathrm{X} 16\}\end{array}\right\}$

$I N D(\{$ Rear right,Rear left $\})=\left\{\begin{array}{l}\{\mathrm{X} 1, \mathrm{X} 2, \mathrm{X} 3, \mathrm{X} 4\},\{\mathrm{X} 7, \mathrm{X} 8, \mathrm{X} 13, \mathrm{X} 14\}, \\ \{\mathrm{X} 5, \mathrm{X} 6, \mathrm{X} 9, \mathrm{X} 10\},\{\mathrm{X} 11, \mathrm{X} 12, \mathrm{X} 15, \mathrm{X} 16\}\end{array}\right\}$ 


$$
\begin{aligned}
& I N D\left(\left\{\begin{array}{l}
\text { front right, } \\
\text { front left, } \\
\text { Rear right }
\end{array}\right\}\right)=\left\{\begin{array}{l}
\{\mathrm{X} 1, \mathrm{X} 7\},\{\mathrm{X} 5, \mathrm{X} 11\},\{\mathrm{X} 3, \mathrm{X} 13\},\{\mathrm{X} 9, \mathrm{X} 15\}, \\
\{\mathrm{X} 2, \mathrm{X} 8\},\{\mathrm{X} 6, \mathrm{X} 12\},\{\mathrm{X} 4, \mathrm{X} 14\},\{\mathrm{X} 10, \mathrm{X} 16\}
\end{array}\right\} \\
& I N D\left(\left\{\begin{array}{l}
\text { front right, } \\
\text { front left, } \\
\text { Rear left }
\end{array}\right\}\right)=\left\{\begin{array}{l}
\{\mathrm{X} 1, \mathrm{X} 5\},\{\mathrm{X} 7, \mathrm{X} 11\},\{\mathrm{X} 3, \mathrm{X} 9\},\{\mathrm{X} 13, \mathrm{X} 15\}, \\
\{\mathrm{X} 2, \mathrm{X} 6\},\{\mathrm{X} 8, \mathrm{X} 12\},\{\mathrm{X} 4, \mathrm{X} 10\},\{\mathrm{X} 14, \mathrm{X} 16\}
\end{array}\right\} \\
& I N D\left(\left\{\begin{array}{l}
\text { front left, } \\
\text { Rear right, } \\
\text { Rear left }
\end{array}\right\}\right)=\left\{\begin{array}{l}
\{\mathrm{X} 1, \mathrm{X} 2\},\{\mathrm{X} 7, \mathrm{X} 8\},\{\mathrm{X} 5, \mathrm{X} 6\},\{\mathrm{X} 11, \mathrm{X} 12\}, \\
\{\mathrm{X} 3, \mathrm{X} 4\},\{\mathrm{X} 13, \mathrm{X} 14\},\{\mathrm{X} 9, \mathrm{X} 10\},\{\mathrm{X} 15, \mathrm{X} 16\}
\end{array}\right\} \\
& I N D\left(\left\{\begin{array}{l}
\text { front right, } \\
\text { front left, } \\
\text { Rear right, } \\
\text { Rear left }
\end{array}\right\}\right)=\left\{\begin{array}{l}
\{\mathrm{x} 1\},\{\mathrm{x} 2\},\{\mathrm{x} 3\},\{\mathrm{x} 4\},\{\mathrm{x} 5\},\{\mathrm{x} 6\},\{\mathrm{x} 7\}, \\
\{\mathrm{X} 8\},\{\mathrm{x} 9\},\{\mathrm{X} 10\},\{\mathrm{X} 11\},\{\mathrm{X} 12\},\{\mathrm{X} 13\}, \\
\{\mathrm{x} 14\},\{\mathrm{x} 15\},\{\mathrm{x} 16\},
\end{array}\right\}
\end{aligned}
$$

pressure data." Proceedings of the Institution of Mechanical Engineers, Part D: Journal of Automobile Engineering 215, no. 1 (2001): 21-29.

[2] Subramanian, Shankar C., Swaroop Darbha, and K. R. Rajagopal. "A diagnostic system for air brakes in commercial vehicles." IEEE transactions on intelligent transportation systems 7, no. 3 (2006): 360-376.

[3] Dhar, Sandeep. Development of diagnostic algorithms for air brakes in trucks. Texas A\&M University, 2010.

[4] Raveendran, Radhika, Apoorva Suresh, Vignesh Rajaram, and Shankar C. Subramanian. "Artificial neural network approach for air brake pushrod stroke prediction in heavy commercial road vehicles." Proceedings of the Institution of Mechanical Engineers, Part D: Journal of Automobile Engineering 233, no. 10 (2019): 2467-2478.

[5] Zhong, N., 2001. A rough sets based knowledge discovery process. International Journal of Applied Mathematics and Computer Science, 11(3), pp.101-117.

[6] Pawlak, Z., 1984, December. On learning — a rough set approach. In Symposium on Computation Theory (pp. 197-227). Springer, Berlin, Heidelberg.

[7] Nabwey, Hossam A. "A Hybrid Approach for Extracting Classification Rules Based on Rough Set Methodology and Fuzzy Inference System and Its Application in Groundwater Quality Assessment." In Advances in Fuzzy Logic and Technology 2017, pp. 611-625. Springer, Cham, 2017.

[8] Nabwey, Hossam A., M. Modather, and M. Abdou. "Rough set theory based method for building knowledge for the rate of heat transfer on free convection over a vertical flat plate embedded in a porous medium." In 2015 International Conference on Computing, Communication and Security (ICCCS), pp. 1-8. IEEE, 2015.

[9] Nabwey, H.A.. An approach based on Rough Sets Theory and Grey System for Implementation of RuleBased Control for Sustainability of Rotary Clinker Kiln. International Journal of Engineering Research and Technology, Volume 12, Number 12 (2019), pp. 2604 2610

[10] Shaaban, Shaaban M., and H. Nabwey. "A decision tree approach for steam turbine-generator fault diagnosis." International Journal of Advanced Science and Technology 51 (2013): 59-66.

[11] Shaaban, Shaaban M., and Hossam A. Nabwey. "A probabilistic rough set approach for water reservoirs site location decision making." In International Conference on Computational Science and Its Applications, pp. 358-372. Springer, Berlin, Heidelberg, 2012.

[12] Shaaban, Shaaban M., and Hossam A. Nabwey. "Rehabilitation and reconstruction of asphalts pavement decision making based on rough set theory."
[1] Kandt, L. D., P. G. Reinhall, and R. R. Scheibe. "Determination of air brake adjustment from air 
In International Conference on Computational Science and Its Applications, pp. 316-330. Springer, Berlin, Heidelberg, 2012.

[13] Shaaban, M., and A. Nabwey. "Transformer fault diagnosis method based on rough set and generalized distribution table." Int J IntellEngSyst 5 (2012): 17-24.

[14] Mohamed, HossamAbdElmaksoud. "An Algorithm for Mining Decision Rules Based on Decision Network and Rough Set Theory." In International Conference on Ubiquitous Computing and Multimedia Applications, pp. 44-54. Springer, Berlin, Heidelberg, 2011.

[15] Zhao, Hong, Ping Wang, Qinghua Hu, and Pengfei Zhu. "Fuzzy Rough Set Based Feature Selection for LargeScale Hierarchical Classification." IEEE Transactions on Fuzzy Systems 27, no. 10 (2019): 1891-1903.

[16] Nabwey, Hossam A., and Mahdy S. El-Paoumy. "An integrated methodology of rough set theory and grey system for extracting decision rules." International Journal of Hybrid Information Technology 6, no. 1 (2013): 57-65.

[17] Pathak, H.K., George, R., Nabwey, H.A., El-Paoumy, M.S. and Reshma, K.P., 2015. Some generalized fixed point results in ab-metric space and application to matrix equations. Fixed Point Theory and Applications, 2015(1), pp.1-17.

[18] George, R., Nabwey, H.A., Reshma, K.P. and Rajagopalan, R., 2015. Generalized cone b-metric spaces and contraction principles. Mat. Vesn, 67(4), pp.246-257.

[19] Nabwey, H.A., Boumazgour, M. and Rashad, A.M., 2017. Group method analysis of mixed convection stagnation-point flow of non-Newtonian nanofluid over a vertical stretching surface. Indian Journal of Physics, 91(7), pp.731-742.

[20] Nabwey, H.A. "An intelligent mining model for medical diagnosis of heart disease based on rough set data analysis". International Journal of Engineering Research and Technology, Volume 13, Number 2 (2020), pp. 355-363
[21] Nabwey, H.A, "Rough set approach for analyzing the effect of viscoelastic and micropolar parameters on hiemenz flow in hydromagnetics". International Journal of Engineering Research and Technology, Volume 13, Number 1 (2020), pp. 170-180

[22] Nabwey, Hossam A., and Hamed A. El-Mky. "Lie group analysis of thermophoresis on a vertical surface in a porous medium." Journal of King Saud UniversityScience 31, no. 4 (2019): 1048-1055.

[23] Nabwey, Hossam A., S. M. M. EL-Kabeir, and A. M. Rashad. "Lie group analysis of effects of radiation and chemical reaction on heat and mass transfer by unsteady slip flow from a non-isothermal stretching sheet immersed in a porous medium." Journal of Computational and Theoretical Nanoscience 12, no. 11 (2015): 4056-4062.

[24] Mohamed, Hossam Abd Elmaksoud. "A probabilistic rough set approach to rule discovery." In International Conference on Ubiquitous Computing and Multimedia Applications, pp. 55-65. Springer, Berlin, Heidelberg, 2011.

[25] Nabwey, H.A. A Mathematical Methodology for Predicting the Primary Site of Metastatic Adenocarcinoma Cancer based on Rough Set Theory. International Journal of Engineering Research and Technology, Volume 13, Number 3 (2020), pp. 427-432

[26] Nabwey, H.A. A Methodology Based on Rough Set Theory and Hypergraph for the Prediction of Wart Treatment. International Journal of Engineering Research and Technology, Volume 13, Number 3 (2020), pp. 552-559

[27] https://engineeringinsider.org/air-brake-systemprinciple-working/

[28] https://www.mechanicalbooster.com/2017/12/air-brakesystem.html

[29] Raveendran, Radhika, K. B. Devika, and Shankar C. Subramanian. "Intelligent Fault Diagnosis of Air Brake System in Heavy Commercial Road Vehicles." In 2020 International Conference on COMmunication Systems \& NETworkS (COMSNETS), pp. 93-98. IEEE, 2020. 
International Journal of Engineering Research and Technology. ISSN 0974-3154, Volume 13, Number 5 (2020), pp. 1002-1008

(C) International Research Publication House. https://dx.doi.org/10.37624/IJERT/13.5.2020.1002-1008

TABLE II. THE UPPER APPROXIMATION AND LOWER APPROXIMATION

\begin{tabular}{|c|c|c|c|c|}
\hline & $\begin{array}{l}\text { Indiscernibility } \\
\text { relation }\end{array}$ & $\begin{array}{l}\text { decision } \\
\text { class }\end{array}$ & The Upper approximation & The Lower approximation \\
\hline 1 & $I N D(\{$ front right $\})$ & \multirow{14}{*}{ 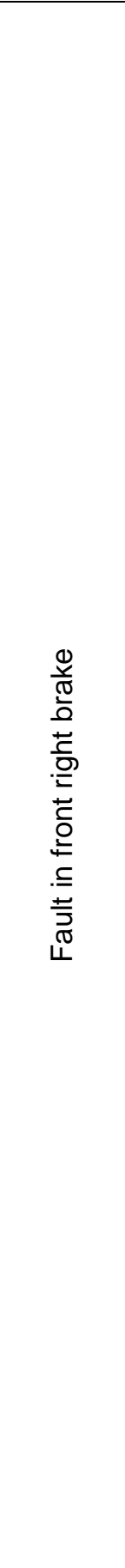 } & $\{\{\mathrm{X} 2, \mathrm{X} 4, \mathrm{X} 6, \mathrm{X} 8, \mathrm{X} 10, \mathrm{X} 12, \mathrm{X} 14, \mathrm{X} 16\}\}$ & $\{\{\mathrm{x} 2, \mathrm{X} 4, \mathrm{X} 6, \mathrm{X} 8, \mathrm{X} 10, \mathrm{X} 12, \mathrm{X} 14, \mathrm{X} 16\}\}$ \\
\hline 2 & $I N D(\{$ front left $\})$ & & $\left\{\begin{array}{l}\{\mathrm{x} 1, \mathrm{X} 2, \mathrm{X} 5, \mathrm{X} 6, \mathrm{X} 7, \mathrm{X} 8, \mathrm{X} 11, \mathrm{X} 12\}, \\
\{\mathrm{X} 3, \mathrm{X} 4, \mathrm{X} 9, \mathrm{X} 10, \mathrm{X} 13, \mathrm{X} 14, \mathrm{X} 15, \mathrm{X} 16\}\end{array}\right\}$ & \\
\hline 3 & $I N D(\{$ Rear right $\})$ & & $\left\{\begin{array}{l}\{\mathrm{x} 1, \mathrm{X} 2, \mathrm{X} 3, \mathrm{X} 4, \mathrm{X} 7, \mathrm{X} 8, \mathrm{X} 13, \mathrm{X} 14\}, \\
\{\mathrm{X} 5, \mathrm{X} 6, \mathrm{X} 9, \mathrm{X} 10, \mathrm{X} 11, \mathrm{X} 12, \mathrm{X} 15, \mathrm{X} 16\}\end{array}\right\}$ & \\
\hline 4 & $I N D(\{$ Rear left $\})$ & & $\left\{\begin{array}{l}\{\mathrm{x} 1, \mathrm{X} 2, \mathrm{X} 3, \mathrm{X} 4, \mathrm{X} 5, \mathrm{X} 6, \mathrm{X} 9, \mathrm{X} 10\}, \\
\{\mathrm{x} 7, \mathrm{x} 8, \mathrm{X} 11, \mathrm{X} 12, \mathrm{X} 13, \mathrm{X} 14, \mathrm{X} 15, \mathrm{X} 16\}\end{array}\right\}$ & \\
\hline 5 & $I N D(\{$ front right,front left $\})$ & & $\{\{\mathrm{x} 2, \mathrm{X} 6, \mathrm{X} 8, \mathrm{X} 12\},\{\mathrm{x} 4, \mathrm{X} 10, \mathrm{X} 14, \mathrm{X} 16\}\}$ & $\{\{\mathrm{x} 2, \mathrm{X} 6, \mathrm{X} 8, \mathrm{X} 12\},\{\mathrm{X} 4, \mathrm{X} 10, \mathrm{X} 14, \mathrm{X} 16\}\}$ \\
\hline 6 & $I N D(\{$ front right,Rear right $\})$ & & $\{\{\mathrm{x} 2, \mathrm{X} 4, \mathrm{X} 8, \mathrm{X} 14\},\{\mathrm{x} 6, \mathrm{X} 10, \mathrm{X} 12, \mathrm{X} 16\}\}$ & $\{\{\mathrm{x} 2, \mathrm{X} 4, \mathrm{X} 8, \mathrm{X} 14\},\{\mathrm{x} 6, \mathrm{X} 10, \mathrm{X} 12, \mathrm{X} 16\}\}$ \\
\hline 7 & $I N D(\{$ front right,Rear left $\})$ & & $\{\{\mathrm{x} 2, \mathrm{X} 4, \mathrm{X} 6, \mathrm{X} 10\},\{\mathrm{x} 8, \mathrm{X} 12, \mathrm{X} 14, \mathrm{X} 16\}\}$ & $\{\{\mathrm{x} 2, \mathrm{X} 4, \mathrm{X} 8, \mathrm{X} 14\},\{\mathrm{x} 6, \mathrm{X} 10, \mathrm{x} 12, \mathrm{x} 16\}\}$ \\
\hline 8 & $I N D(\{$ front left,Rear right $\})$ & & $\left\{\begin{array}{l}\{\mathrm{x} 1, \mathrm{x} 2, \mathrm{x} 7, \mathrm{x} 8\},\{\mathrm{x} 5, \mathrm{x} 6, \mathrm{X} 11, \mathrm{X} 12\}, \\
\{\mathrm{x} 3, \mathrm{X} 4, \mathrm{x} 13, \mathrm{X} 14\},\{\mathrm{x} 9, \mathrm{X} 10, \mathrm{x} 15, \mathrm{x} 16\}\end{array}\right\}$ & \\
\hline 9 & $I N D(\{$ front left,Rear left $\})$ & & $\left\{\begin{array}{l}\{\mathrm{x} 1, \mathrm{x} 2, \mathrm{x} 5, \mathrm{x} 6\},\{\mathrm{x} 7, \mathrm{x} 8, \mathrm{X} 11, \mathrm{x} 12\}, \\
\{\mathrm{x} 3, \mathrm{x} 4, \mathrm{x} 9, \mathrm{x} 10\},\{\mathrm{x} 13, \mathrm{X} 14, \mathrm{X} 15, \mathrm{x} 16\}\end{array}\right\}$ & \\
\hline 10 & $I N D(\{$ Rear right,Rear left $\})$ & & $\left\{\begin{array}{l}\{\mathrm{x} 1, \mathrm{X} 2, \mathrm{X} 3, \mathrm{x} 4\},\{\mathrm{x} 7, \mathrm{x} 8, \mathrm{X} 13, \mathrm{X} 14\}, \\
\{\mathrm{x} 5, \mathrm{X} 6, \mathrm{X} 9, \mathrm{X} 10\},\{\mathrm{x} 11, \mathrm{X} 12, \mathrm{X} 15, \mathrm{X} 16\}\end{array}\right\}$ & \\
\hline 11 & $I N D\left(\left\{\begin{array}{l}\text { front right, } \\
\text { front left, } \\
\text { Rear right }\end{array}\right\}\right)$ & & $\{\{\mathrm{X} 2, \mathrm{X} 8\},\{\mathrm{x} 6, \mathrm{X} 12\},\{\mathrm{x} 4, \mathrm{X} 14\},\{\mathrm{x} 10, \mathrm{X} 16\}\}$ & $\{\{\mathrm{x} 2, \mathrm{x} 8\},\{\mathrm{x} 6, \mathrm{x} 12\},\{\mathrm{x} 4, \mathrm{X} 14\},\{\mathrm{x} 10, \mathrm{X} 16\}\}$ \\
\hline 12 & $I N D\left(\left\{\begin{array}{l}\text { front right, } \\
\text { front left, } \\
\text { Rear left }\end{array}\right\}\right)$ & & $\{\{\mathrm{x} 2, \mathrm{x} 6\},\{\mathrm{x} 8, \mathrm{X} 12\},\{\mathrm{x} 4, \mathrm{X} 10\},\{\mathrm{x} 14, \mathrm{X} 16\}\}$ & $\{\{\mathrm{x} 2, \mathrm{x} 6\},\{\mathrm{x} 8, \mathrm{x} 12\},\{\mathrm{x} 4, \mathrm{x} 10\},\{\mathrm{x} 14, \mathrm{X} 16\}\}$ \\
\hline 13 & $I N D\left(\left\{\begin{array}{l}\text { front left, } \\
\text { Rear right, } \\
\text { Rear left }\end{array}\right\}\right)$ & & $\left\{\begin{array}{l}\{\mathrm{x} 1, \mathrm{x} 2\},\{\mathrm{x} 7, \mathrm{x} 8\},\{\mathrm{x} 5, \mathrm{x} 6\},\{\mathrm{x} 11, \mathrm{x} 12\} \\
\{\mathrm{x} 3, \mathrm{x} 4\},\{\mathrm{x} 13, \mathrm{x} 14\},\{\mathrm{x} 9, \mathrm{x} 10\},\{\mathrm{x} 15, \mathrm{x} 16\}\end{array}\right\}$ & \\
\hline 14 & $I N D\left(\left\{\begin{array}{l}\text { front right, front left, } \\
\text { Rear right, Rear left }\end{array}\right\}\right)$ & & $\left\{\begin{array}{l}\{\mathrm{x} 2\},\{\mathrm{x} 8\},\{\mathrm{x} 6\},\{\mathrm{x} 12\}, \\
\{\mathrm{x} 4\},\{\mathrm{x} 14\},\{\mathrm{x} 10\},\{\mathrm{x} 16\}\end{array}\right\}$ & \\
\hline
\end{tabular}


International Journal of Engineering Research and Technology. ISSN 0974-3154, Volume 13, Number 5 (2020), pp. 1002-1008

(C) International Research Publication House. https://dx.doi.org/10.37624/IJERT/13.5.2020.1002-1008

TABLE III. THE GENERATED RULES FOR CLASSIFYING THE “FAULT” AND "NO-FAULT" CONDITIONS OF AIR BRAKE SYSTEM

\begin{tabular}{|c|c|c|c|c|}
\hline & Rule & $\begin{array}{l}\text { LHS } \\
\text { SUPPORT }\end{array}$ & $\begin{array}{c}\text { RHS } \\
\text { SUPPORT }\end{array}$ & $\begin{array}{c}\text { RHS } \\
\text { ACCURACY } \\
\end{array}$ \\
\hline 1 & $\begin{array}{l}\text { front right(No) AND front left(No) AND Rear right(No) AND Rear left(No) => } \\
\text { Decision (No Fault in front right brake) OR Decision (No Fault in front left brake) }\end{array}$ & 2 & 1,1 & $0.5,0.5$ \\
\hline 2 & $\begin{array}{l}\text { front right(Yes) AND front left(No) AND Rear right(No) AND Rear left(No) => } \\
\text { Decision (Fault in front right brake) OR Decision (No Fault in front left brake) }\end{array}$ & 2 & 1,1 & $0.5,0.5$ \\
\hline 3 & $\begin{array}{l}\text { front right(No) AND front left(Yes) AND Rear right(No) AND Rear left(No) }=> \\
\text { Decision (No Fault in front right brake) OR Decision (Fault in frontleft brake) }\end{array}$ & 2 & 1,1 & $0.5,0.5$ \\
\hline 4 & $\begin{array}{l}\text { front right }(*) \text { AND front left(Yes) AND Rear right }(*) \text { AND Rear left(No) } \Rightarrow> \\
\text { Decision (Fault in front right brake) }\end{array}$ & 1 & 1 & 1.0 \\
\hline 5 & $\begin{array}{l}\text { front right(No) AND front left(No) AND Rear right(Yes) AND Rear left(No) }=> \\
\text { Decision (No Fault in front right brake) }\end{array}$ & 1 & 1 & 1.0 \\
\hline 6 & $\begin{array}{l}\text { front right(Yes) AND front left(*) AND Rear right(Yes) AND Rear left(No) }=> \\
\text { Decision (Fault in front right brake) OR Decision (No Fault in front left brake) }\end{array}$ & 2 & 1,1 & $0.5,0.5$ \\
\hline 7 & $\begin{array}{l}\text { front right(No) AND front left(No) AND Rear right(No) AND Rear left(*) } \Rightarrow> \\
\text { Decision (No Fault in front right brake) }\end{array}$ & 1 & 1 & 1.0 \\
\hline 8 & $\begin{array}{l}\text { front right(Yes) AND front left(No) AND Rear right(*) AND Rear left(Yes) }=> \\
\text { Decision (Fault in front right brake) }\end{array}$ & 1 & 1 & 1.0 \\
\hline 9 & $\begin{array}{l}\text { front right(No) AND front left(Yes) AND Rear right(Yes) AND Rear left(No) => } \\
\text { Decision (No Fault in front right brake) OR Decision (Fault in frontleft brake) }\end{array}$ & 2 & 1,1 & $0.5,0.5$ \\
\hline 10 & $\begin{array}{l}\text { front right(Yes) AND front left(Yes) AND Rear right(*) AND Rear left(No) }=> \\
\text { Decision (Fault in front right brake) }\end{array}$ & 1 & 1 & 1.0 \\
\hline 11 & $\begin{array}{l}\text { front right(No) AND front left(No) AND Rear right(Yes) AND Rear left(Yes) } \Rightarrow> \\
\text { Decision (No Fault in front right brake) OR Decision (No Fault in front left brake) }\end{array}$ & 2 & 1,1 & $0.5,0.5$ \\
\hline 12 & $\begin{array}{l}\text { front right(*) AND front left(No) AND Rear right(Yes) AND Rear left(Yes) }=> \\
\text { Decision (Fault in front right brake) }\end{array}$ & 1 & 1 & 1.0 \\
\hline 13 & $\begin{array}{l}\text { front right(No) AND front left(Yes) AND Rear right(No) AND Rear left(*) } \Rightarrow> \\
\text { Decision (No Fault in front right brake) }\end{array}$ & 1 & 1 & 1.0 \\
\hline 14 & $\begin{array}{l}\text { front right(Yes) AND front left(Yes) AND Rear right(No) AND Rear left(Yes) }=> \\
\text { Decision (Fault in front right brake) }\end{array}$ & 1 & 1 & 1.0 \\
\hline 15 & $\begin{array}{l}\text { front right(No) AND front left(Yes) AND Rear right(Yes) AND Rear left(Yes) => } \\
\text { Decision (No Fault in front right brake) OR Decision (Fault in frontleft brake) }\end{array}$ & 2 & 1,1 & $0.5,0.5$ \\
\hline 16 & $\begin{array}{l}\text { front right(Yes) AND front left(*) AND Rear right(Yes) AND Rear left(Yes) => } \\
\text { Decision (Fault in front right brake) }\end{array}$ & 1 & 1 & 1.0 \\
\hline 17 & $\begin{array}{l}\text { front right(*) AND front left(*) AND Rear right(No) AND Rear left(No) }=> \\
\text { Decision (Fault in frontleft brake) }\end{array}$ & 1 & 1 & 1.0 \\
\hline 18 & $\begin{array}{l}\text { front right(No) AND front left(No) AND Rear right(*) AND Rear left(Yes) } \Rightarrow> \\
\text { Decision (No Fault in front left brake) }\end{array}$ & 1 & 1 & 1.0 \\
\hline 19 & $\begin{array}{l}\text { front right(No) AND front left(Yes) AND Rear right(No) AND Rear left(Yes) => } \\
\text { Decision (Fault in frontleft brake) }\end{array}$ & 1 & 1 & 1.0 \\
\hline 20 & $\begin{array}{l}\text { front right(No) AND front left(*) AND Rear right(Yes) AND Rear left(*) } \Rightarrow \\
\text { Decision (No Fault in front left brake) }\end{array}$ & 1 & 1 & 1.0 \\
\hline 21 & $\begin{array}{l}\text { front right }(*) \text { AND front left(No) AND Rear right }(*) \text { AND Rear left(Yes) } \Rightarrow> \\
\text { Decision (No Fault in front left brake) }\end{array}$ & 1 & 1 & 1.0 \\
\hline 22 & $\begin{array}{l}\text { front right(Yes) AND front left(Yes) AND Rear right(*) AND Rear left(Yes) => } \\
\text { Decision (Fault in frontleft brake) }\end{array}$ & 1 & 1 & 1.0 \\
\hline 23 & $\begin{array}{l}\text { front right(*) AND front left(Yes) AND Rear right(Yes) AND Rear left(No) }=> \\
\text { Decision (Fault in frontleft brake) }\end{array}$ & 1 & 1 & 1.0 \\
\hline 24 & $\begin{array}{l}\text { front right(Yes) AND front left(No) AND Rear right(Yes) AND Rear left(*) } \Rightarrow> \\
\text { Decision (No Fault in front left brake) }\end{array}$ & 1 & 1 & 1.0 \\
\hline 25 & $\begin{array}{l}\text { front right(*) AND front left(Yes) AND Rear right(*) AND Rear left(Yes) }=> \\
\text { Decision (Fault in frontleft brake) }\end{array}$ & 1 & 1 & 1.0 \\
\hline
\end{tabular}

